\title{
DISTRIBUIÇÃO ESPAÇO-TEMPORAL DE LARVAS DE SCIAENIDAE EM UM ESTUÁRIO SUBTROPICAL (SANTA CATARINA, BRASIL)
}

\author{
COSTA, M. D. P. ${ }^{1 *}$; SCHWINGEL, P. R. ${ }^{2}$; SOUZA-CONCEIÇÃO, J. M. ${ }^{3} \&$ SPACH, H. L. ${ }^{4}$ \\ 1 - Laboratório de Ecologia do Ictioplâncton (Instituto de Oceanografia), Universidade Federal do Rio \\ Grande - FURG \\ 2 - Laboratório de Oceanografia Biológica, Universidade do Vale do Itajaí - UNIVALI \\ 3 - EEB Alexandre Guilherme Figueredo - SED- SC \\ 4 - Centro de Estudos do Mar, Universidade Federal do Paraná - UFPR \\ ${ }^{*}$ Contact author: duarte.micheli@yahoo.com.br
}

\begin{abstract}
Costa, M. D. P.; Schwingel, P. R.; Souza-Conceição, J. M. \& Spach, H. L. (2012) Spatio-temporal distribution of Sciaenidae larvae in a subtropical estuary (Santa Catarina, Brazil). Braz. J. Aquat. Sci. Technol. 16(2): 51-59. eISSN 1983-9057. This work studied the use of the Babitonga Bay $\left(26^{\circ} 02^{\prime}-26^{\circ} 28^{\prime} S\right.$ and $\left.48^{\circ} 28^{\prime}-48^{\circ} 50^{\prime} \mathrm{W}\right)$ by early life stages of Sciaenidae, considering the spatial-temporal distribution of species and their relationship with environmental factors. Samples were obtained at nine stations between October 2007 and August 2008, with 2 minutes oblique tows using a $40 \mathrm{~cm}$ diameter conical plankton net fitted with $200 \mu \mathrm{m}$ mesh and 2 five minutes oblique tows with a $50 \mathrm{~cm}$ diameter cylindrical-conical plankton net fitted with $500 \mu \mathrm{m}$ mesh. A total of 249 fish larvae of Sciaenidae were caught, with Cynoscion spp. (50\%), Umbrina spp. (16\%) and Stellifer spp. (17,6\%) being the most abundant taxa. Fish larvae showed the highest densities during Spring and Summer months, and occupied the whole estuary. Temperature and salinity were the main factors structuring the distribution of Sciaenidae larvae. Preflexion stage was the most abundant, but vitelline, flexion and postflexion larvae were also reported. The presence of different early life stages of Sciaenidae in Babitonga Bay reveals the important role of this ecosystem as a nursery for target species of South-Southeast Brazil fisheries.
\end{abstract}

Key words: ichthyoplankton, Babitonga Bay, Sciaenidae, Cynoscion spp., Umbrina spp., Micropogonias furnieri

\section{INTRODUÇÃO}

A família Sciaenidae compreende peixes teleósteos que habitam águas marinhas, salobras e fluviais, com cerca de 270 espécies distribuídas em 70 gêneros (Nelson, 2006). A maioria das espécies habita águas rasas da plataforma continental, mas dependem de ambientes estuarinos em diferentes períodos do ciclo de vida. A atividade pesqueira sobre espécies desta família é importante em várias partes do mundo (Luczkovich et al., 2008), inclusive na região sudestesul do Brasil, onde Micropogonias furnieri (Desmarest, 1823) (corvina), Cynoscion guatucupa (Cuvier, 1830) (pescada), Cynoscion jamaicensis (Vaillant \& Bocourt, 1883) (goete) e Macrodon atricauda (Günther, 1880) (pescadinha-real) são espécies-alvo da pesca (Haimovici, 1998; Cergole et al. 2005).

As larvas de peixes podem utilizar uma ampla variedade de habitats, desde a plataforma continental até lagoas costeiras, baías e estuários. Os estuários disponibilizam para os estágios iniciais de peixes uma grande gama de habitats durante todo o seu desenvolvimento, incluindo marismas, manguezais, enseadas rasas, áreas rochosas, fundo não vegetado (arenoso, areno-lodoso e lodoso) e a própria coluna d'água. Impactos na estrutura destes habitats podem influenciar a produção pesqueira, devido à associação entre a sobrevivência dos estágios iniciais e abundância de espécies comercialmente exploradas (Hoss \& Thayer, 1993). Babler (2000) descreve que todos os parâmetros físicos, aos quais as larvas de peixes estão submetidas nesses ambientes, são capazes de afetar o recrutamento, podendo influenciar na distribuição, ocorrência e composição de espécies.

Estudos revelam a importância dos estuários como área de alimentação e berçário para os estágios iniciais de desenvolvimento de espécies da família Sciaenidae na costa brasileira (Sinque, 1980; Sinque, 1989; Muelbert \& Weiss, 1991; Barletta-Bergan et al., 2002; Coser et al., 2007; Bonecker et al., 2009; Costa \& Souza-Conceição, 2009). Assim, os estuários são considerados áreas-chave para o desenvolvimento de espécies de interesse econômico, sendo a conservação destes ambientes fundamental para a manutenção de várias espécies marinhas (Lenanton \& Potter, 1987).

A baía da Babitonga está localizada no norte do Estado de Santa Catarina (Brasil) e comporta, em termos latitudinais, a última grande formação de manguezal do hemisfério sul. Estudos anteriores evidenciam a importância deste ecossistema para os estágios iniciais das diferentes espécies de peixes, juvenis e adultos (IBAMA, 1998; Côrrea et al., 2006; Souza-Conceição, 2008; Costa \& Souza-Conceição, 
2009; Costa et al., 2011), incluindo diversos representantes da família Sciaenidae. A miraguaia (Pogonias cromis Linnaeus, 1766), a corvina (Micropogonias furnieri) e as pescadas (Cynoscion spp.) constituem importantes recursos pesqueiros para a atividade artesanal da região (Bastos, 2006).

O presente estudo teve como objetivo avaliar o uso do estuário da baía da Babitonga pelas larvas da família Sciaenidae, considerando a distribuição espaço-temporal dos taxa e sua relação com parâmetros abióticos e bióticos. Os resultados fornecerão subsídios para compreender qual o papel do ecossistema da baía da Babitonga para o desenvolvimento dos estágios iniciais do ciclo de vida da família Sciaenidae.

\section{MATERIAIS E MÉTODOS}

Oito coletas de ictioplâncton foram realizadas em 9 pontos amostrais no canal da baía da Babitonga

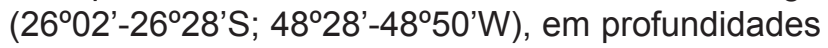
de 3 a 20 metros, entre outubro de 2007 e agosto de 2008. Os pontos foram distribuídos no canal do estuário, incluindo pontos no setor externo (pontos amostrais 1, 2 e 3), setor intermediário (4, 5 e 6) e setor interno (7, 8 e 9) (Figura 1). As amostras foram coletadas com dois modelos de rede: 1 ) rede cônica de $200 \mu \mathrm{m}$ de abertura de malha, $40 \mathrm{~cm}$ de diâmetro de boca e 1,4 m de comprimento e 2) rede cilíndrico-cônica de $500 \mu \mathrm{m}$ de abertura de malha, $50 \mathrm{~cm}$ de diâmetro de boca e 2,5 m de comprimento. As redes foram equipadas com fluxômetro, sendo que os arrastos foram oblíquos, com duração de 2 min com a rede de $200 \mu \mathrm{m}$ e $5 \mathrm{~min}$ com a rede de $500 \mu \mathrm{m}$. A rede de $200 \mu \mathrm{m}$ foi utilizada para abranger os estágios de desenvolvimento de menores classes de tamanho, enquanto que a rede de $500 \mu \mathrm{m}$ para a coleta de indivíduos maiores. Todas as amostras foram fixadas com solução formalina $4 \%$.

Dados de temperatura e salinidade (superfície e fundo) foram registrados para cada ponto amostral com um multianalisador Horiba Modelo U10. A transparência foi determinada em centímetros com um disco de Secchi, e a clorofila a, a partir de uma alíquota de água filtrada de superfície com filtro de fibra de vidro $(25 \mathrm{~mm}$ de diâmetro e 1,2 $\mu \mathrm{m}$ de abertura de poro). Os filtros foram mantidos em papel alumínio, etiquetados, armazenados e resfriados para análise em laboratório. Os pigmentos de clorofila a foram extraídos das amostras com acetona $90 \%$ durante 24 horas no escuro a $12^{\circ} \mathrm{C}$. Em seguida foi realizada análise da concentração $(\mu \mathrm{g} / \mathrm{L})$ em fluorímetro Turner Designs modelo Trilogy (Machado et al., 1997; Diaz et al., 2000).

Em laboratório, as larvas de Sciaenidae foram triadas e identificadas ao menor nível taxonômico possível, sob microscópio estereoscópico binocular, de acordo com referências bibliográficas especializadas (Johnson, 1978; Matsuura \& Nakatani, 1979; Menezes \& Figueiredo, 1980; Sinque, 1980; Fahay,
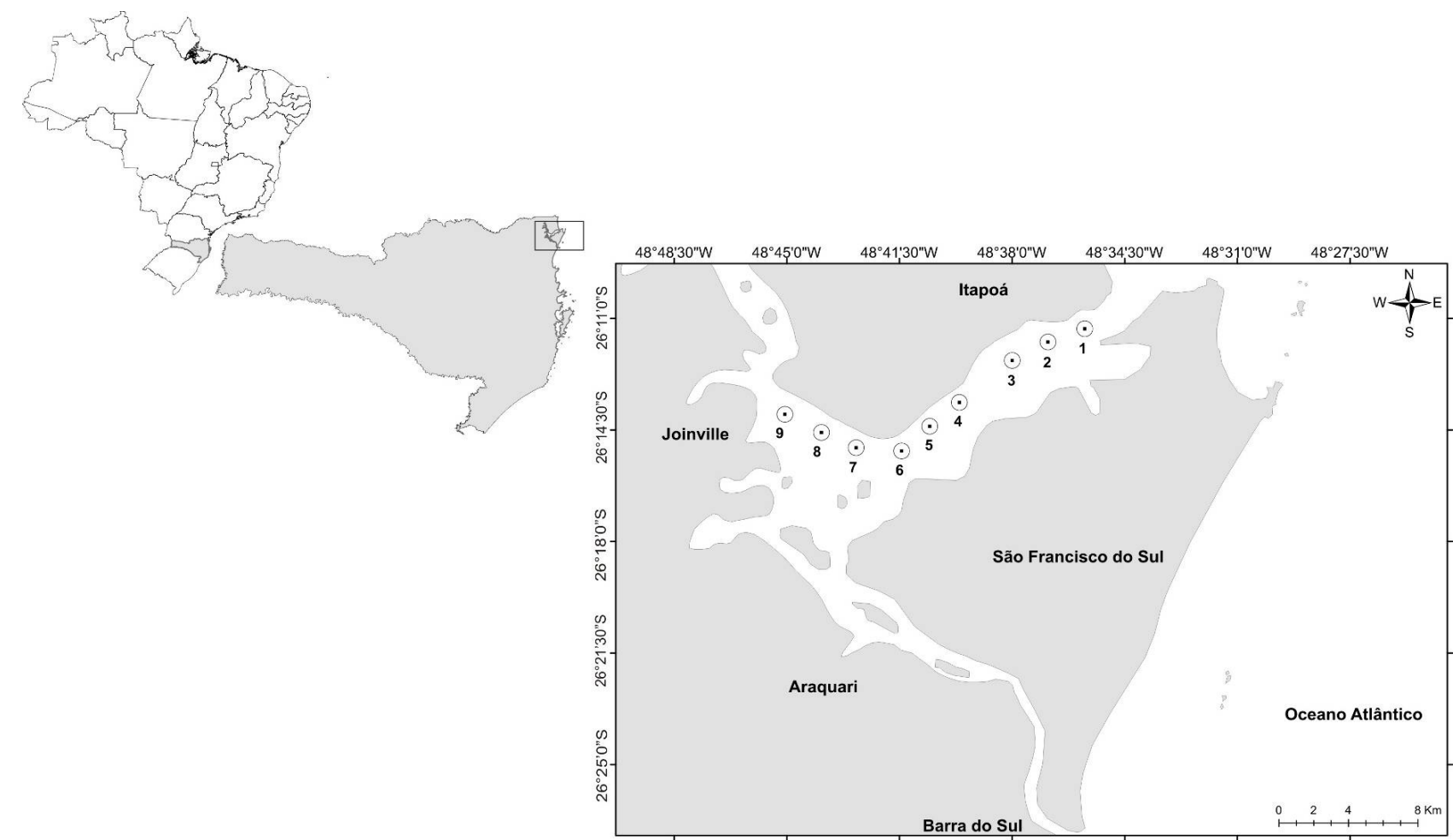

Figura 1 - Localização da baía da Babitonga (Santa Catarina, Brasil) e posição dos nove pontos amostrais. 
1983; Leis \& Rennis, 1983; Ditty, 1989; Leis \& Trnski, 1989; Olivar \& Fortuño, 1991; Moser, 1996; Ré, 1999; Richards, 2006; e Itagaki et al., 2007), sendo então calculadas as abundâncias de larvas por cem metros cúbicos $\left(\mathrm{n}^{\circ} \times 100 \mathrm{~m}^{-3}\right)$. Os estágios de desenvolvimento foram determinados como vitelínico, pré-flexão, flexão e pós-flexão, sendo utilizada régua micrométrica (precisão: 0,1mm) para medidas de comprimento da notocorda (estágios: vitelínico, pré-flexão e flexão) e de comprimento padrão (estágio: pós-flexão) para todas as larvas coletadas (Richards, 2006).

Para verificar diferenças significativas $(p<0,05)$ na variação dos parâmetros de temperatura, salinidade, transparência e clorofila a entre os meses, superfície e fundo, e os pontos de coleta aplicou-se ANOVA e havendo significância, foi usado o teste de Tuckey. Para os dados que não apresentaram homocedasticidade de variâncias, testada através do teste de Bartlett $(p<0,05)$, aplicou-se alternativamente os testes não-paramétricos de Kruskal-Wallis e Dunn (Zar, 1996).

A análise da relação entre as abundâncias de larvas de Sciaenidae e os parâmetros abióticos e bióticos foi realizada através do procedimento BIO-ENV do programa PRIMER 6.0, sendo que este se baseia na comparação entre matrizes de similaridade, resultando em índices de correlação de Spearman para cada combinação possível entre as variáveis (Clarke \& Gorley, 2006). Neste procedimento foram utilizados os parâmetros de densidade larval, temperatura, salinidade, transparência da água e concentração de clorofila $a$.

\section{RESULTADOS}

\section{Condições ambientais na baía da Babitonga ao longo do período de estudo}

A temperatura da água teve variação significativa $(p<0,05)$ entre os meses de coleta, oscilando entre $19^{\circ} \mathrm{C}$ em agosto e $26^{\circ} \mathrm{C}$ em janeiro (Figura 2a). Ao longo dos pontos amostrais foi observado aumento da temperatura no setor interno da baía da Babitonga (Figura 2b), entretanto esse padrão não mostrou significância $(p>0,05)$. Em relação a coluna d'água, não foram verificadas variações significativas de temperatura $(p>0,05)$, apesar de valores mais elevados serem registrados na superfície.

Com relação a salinidade, foram observadas variações significativas $(p<0,05)$ entre os meses de coleta, com valores menores sendo registrados em fevereiro (18) e os maiores em junho (36) (Figura 2c). Em relação aos pontos amostrais, foi observado um gradiente decrescente significativo $(p<0,05)$ do setor externo para o interno do estuário (Figura $2 \mathrm{~d}$ ).
A salinidade não foi diferente entre a superfície e o fundo $(p>0,05)$.

A transparência da coluna d'água foi maior nos pontos amostrais de maiores profundidades, ou seja, naqueles mais próximos a barra do estuário (setor externo) (Figura 2f). Entre os meses, foram registrados maiores valores de transparência em abril e julho de 2008 (Figura 2e), onde foram constatadas variações significativas na transparência da água $(p<0,05)$ entre os meses de coleta.

A concentração de clorofila a foi semelhante entre os pontos amostrais, com valores médios entre $8-17 \mu \mathrm{g} / \mathrm{L}$, porém esta variação não foi significativa $(p>0,05)$ (Figura 2h). Entre os meses de coleta (Figura $2 g)$ foi possível observar variação significativa $(p<0,05)$ da clorofila a, ocorrendo maiores concentrações nos meses de verão (janeiro e fevereiro de 2008) e julho de 2008, com decréscimo em agosto de 2008.

\section{Diversidade de larvas e sua variação espaço-temporal}

Um total de 5420 larvas de peixes foi coletado, das quais 3380 com a rede de plâncton cônica de $200 \mu \mathrm{m}$ e 2040 com a cilíndrico-cônica de $500 \mu \mathrm{m}$. Destas, 249 larvas foram identificadas como Sciaenidae, 165 coletadas com a rede de $200 \mu \mathrm{m}$ e 84 com a rede de $500 \mu \mathrm{m}$. Durante o período de estudo foi possível identificar nove taxa (Tabela 1), sendo os mais abundantes Cynoscion spp. $\left(102,1 \times 100 \mathrm{~m}^{-3} \mathrm{com}\right.$ a rede de $200 \mu \mathrm{m}$ e $3,1 \times 100 \mathrm{~m}^{-3} \mathrm{com}$ a de $500 \mu \mathrm{m}$ ), Umbrina spp. $\left(45,29 \times 100 \mathrm{~m}^{-3} \mathrm{com}\right.$ a rede de $\left.200 \mu \mathrm{m}\right)$ e Stellifer spp. $\left(15,48 \times 100 \mathrm{~m}^{-3} \mathrm{com}\right.$ a rede de $200 \mu \mathrm{m}$ e $3,87 \times 100 \mathrm{~m}^{-3} \mathrm{com}$ a de $\left.500 \mu \mathrm{m}\right)$. Os taxa Sciaenidae (não identificados, $\mathrm{NI}$ ), Isopisthus parvipinnis e Umbrina spp. ocorreram somente nas amostras da rede de $200 \mu \mathrm{m}$, enquanto que Larimus breviceps apenas na rede de $500 \mu \mathrm{m}$.

As larvas de Sciaenidae foram registradas em todos os setores do estuário, sendo que Cynoscion spp. foi o único taxon encontrado em todos os pontos amostrais. Sciaenidae NI, Bairdiella ronchus, Micropogonias furnieri, Stellifer spp. e Umbrina spp. também ocuparam diferentes setores da baía da Babitonga. Por outro lado, I. parvipinnis e L. breviceps mostraram baixa abundância e ocorrência restrita ao setor externo, enquanto que Macrodon atricauda foi identificado no setor externo e intermediário (Tabela 1). Com relação a variação temporal, a maior abundância de larvas esteve relacionada com os meses de primavera e verão (Tabela 1). Este padrão foi marcante para Cynoscion spp. e também foi observado para Umbrina spp., Stellifer spp. e B. ronchus, apesar da menor abundância (Tabela 1). 

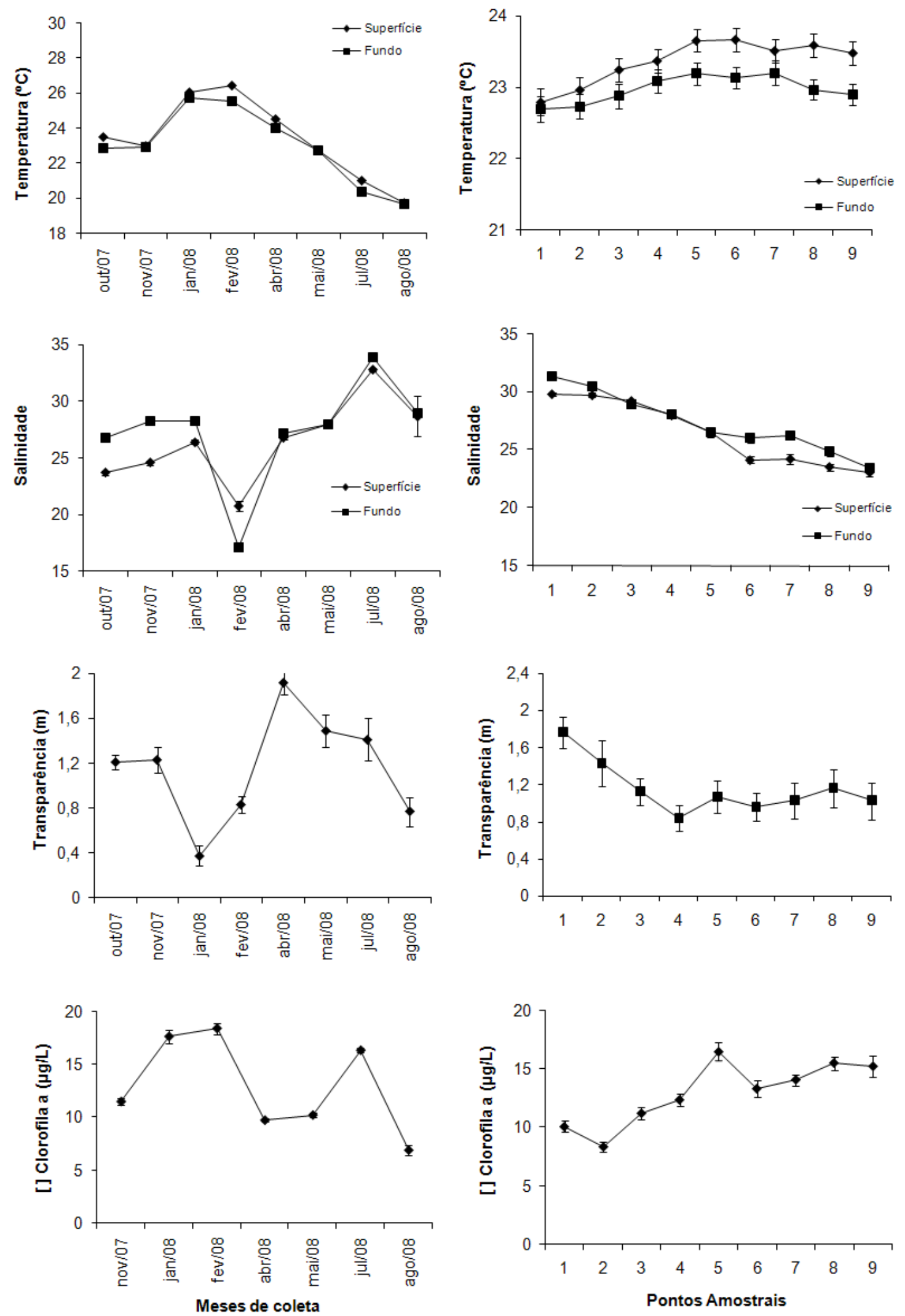

Figura 2 - Variação mensal e espacial (média \pm erro padrão) da temperatura, salinidade, transparência e clorofila a superficial, durante o período de outubro de 2007 a agosto de 2008, na baía da Babitonga. 


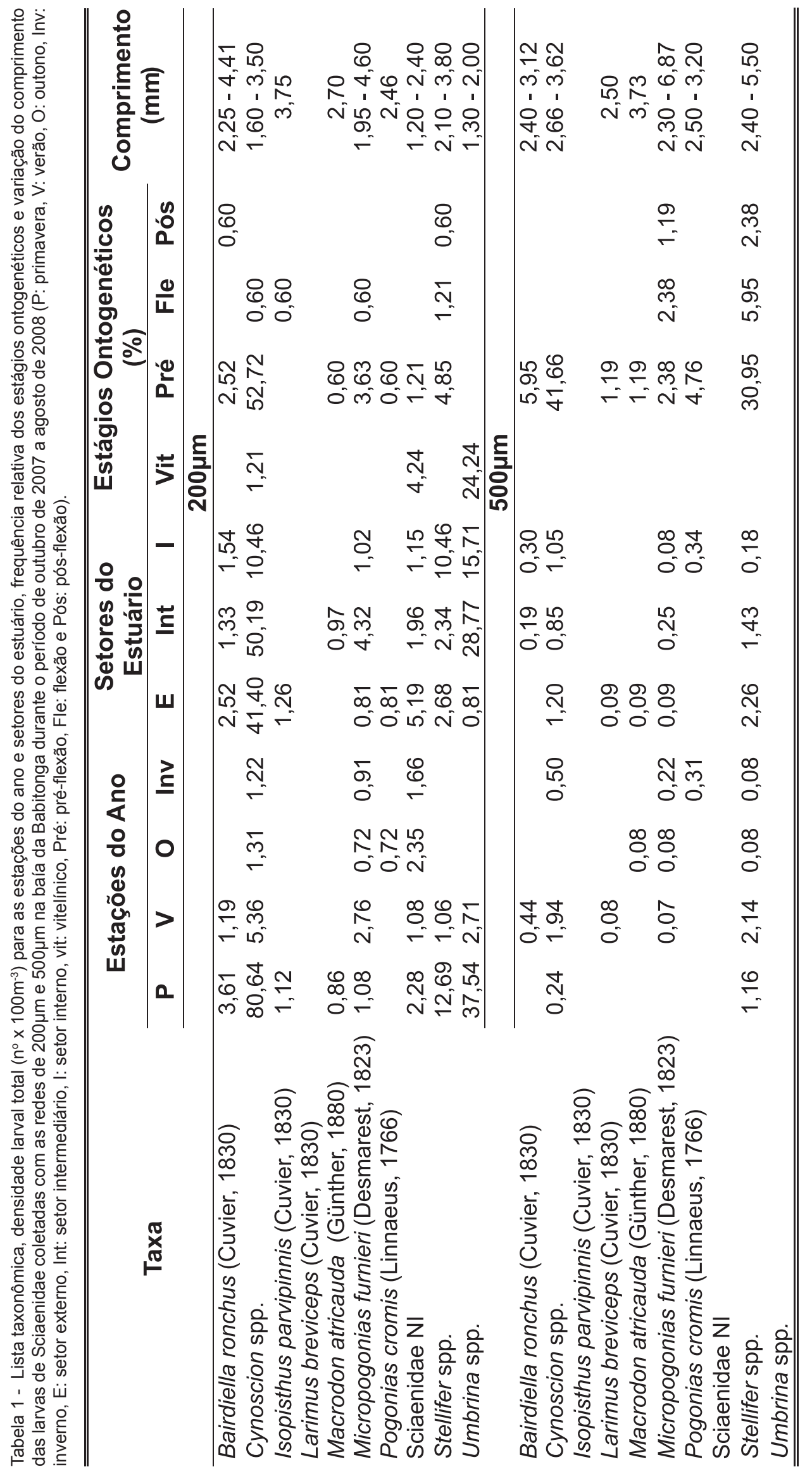


Tabela 2 - Resultado de correlações do procedimento BIO-ENV entre as variáveis abióticas e bióticas (temperatura, salinidade, transparência e concentração de clorofila a) e as abundâncias das larvas de Sciaenidae obtidas com as redes de plâncton de $200 \mu \mathrm{m}$ e $500 \mu \mathrm{m}$. Nota: em negrito as melhores correlações.

\begin{tabular}{lcc}
\hline \hline \multirow{2}{*}{ Variáveis } & \multicolumn{2}{c}{$\begin{array}{c}\text { Coeficiente de Correlação } \\
\text { de Spearman }\end{array}$} \\
\cline { 2 - 3 } & $\mathbf{2 0 0} \boldsymbol{\mu m}$ & $\mathbf{5 0 0} \boldsymbol{\mu m}$ \\
\hline Temperatura + Salinidade & $\mathbf{0 , 5 3}$ & 0,45 \\
Temperatura & $\mathbf{0 , 5 2}$ & - \\
Salinidade + Transparência + Clorofila a & - & $\mathbf{0 , 6 2}$ \\
Temperatura + Salinidade + Transparência & 0,42 & $\mathbf{0 , 5 7}$ \\
Todas as variáveis & 0,41 & 0,56 \\
Temperatura + Salinidade + Clorofila a & 0,48 & - \\
Salinidade & 0,45 & 0,55 \\
Salinidade + Clorofila a & 0,45 & 0,52 \\
Temperatura + Transparência + Clorofila a & 0,39 & 0,50 \\
\hline \hline
\end{tabular}

\section{Estágios de desenvolvimento}

As larvas no estágio de desenvolvimento de pré-flexão (66\%) foram as mais abundantes em ambas as redes utilizadas (Tabela 1). O estágio vitelínico foi registrado apenas com a rede de menor abertura de malha $(200 \mu \mathrm{m})$, enquanto os demais estágios foram coletados por ambas as redes $(200 \mu \mathrm{m}$ e $500 \mu \mathrm{m})$. Umbrina spp. ocorreu somente no estágio vitelínico, com tamanhos entre 1,30-2,00mm, e $B$. ronchus em estágios de pré-flexão e pós-flexão com 2,25 $4,41 \mathrm{~mm}$ de comprimento. Para Cynoscion spp. foram registradas larvas entre 1,60 a $3,62 \mathrm{~mm}$, com maior abundância relativa de indivíduos no estágio de pré-flexão. Os taxa M. furnieri $(1,95-6,87 \mathrm{~mm})$ e Stellifer spp. $(2,10-5,50 \mathrm{~mm})$ apareceram nas amostras nos estágios de pré-flexão, em flexão e pós-flexão, sendo que indivíduos em pré-flexão foram os mais abundantes (Tabela 1).

\section{Relação entre larvas de Sciaenidae e os parâmetros abióticos e bióticos}

O resultado do procedimento BIO-ENV (Tabela 2), entre as larvas de Sciaenidae amostradas com a rede de $200 \mu \mathrm{m}$ e as demais variáveis, revelou que a combinação que melhor explica o padrão de distribuição das larvas é temperatura e salinidade $(\rho=0,53)$, seguida pela temperatura $(\rho=0,52)$. Com relação a distribuição das larvas coletadas com a rede de $500 \mu \mathrm{m}$, a combinação de variáveis que melhor explica o padrão de distribuição é salinidade, transparência e clorofila a $(\rho=0,62)$, seguida pela combinação temperatura, salinidade e transparência $(\rho=0,57)$.

\section{DISCUSSÃO}

No estuário da baía da Babitonga não foi observado variação significativa de temperatura e salinidade entre os estratos da coluna d'água, o que confirma a condição de estuário verticalmente homogêneo existente na área (Oliveira et al., 2006; Camacho \& Souza-Conceição, 2007; Costa \& Souza-Conceição, 2009). Porém, foi observado um gradiente horizontal, com diminuição da salinidade e transparência entre a área externa e interna, padrão já observado em outros estudos (Oliveira et al., 2006; Camacho \& Souza-Conceição, 2007). Além disso, os resultados dos fatores físicos, químicos e biológicos em estuários mostram que estes passam a ser selecionados ativamente ao longo dos estágios iniciais de desenvolvimento dos peixes (Boehlert \& Mundy, 1988; Elliott \& Hemingway, 2002), o que explica as combinações de mais variáveis sobre as larvas amostradas com a rede de $500 \mu \mathrm{m}$, composta por organismos de maiores tamanhos.

A utilização espaço-temporal de toda a baía da Babitonga pelos estágios iniciais de Sciaenidae foi evidente, principalmente pelos taxa Cynoscion spp., Stellifer spp. e Umbrina spp. A coleta somente de estágio vitelínico de Umbrina spp. indica a proximidade da área de desova desta espécie no estuário da Babitonga e/ou área costeira adjacente. Além disso, não se pode descartar a possibilidade de que as larvas mais desenvolvidas de Umbrina spp. ocupem áreas não contempladas na amostragem, o que pode direcionar pesquisas futuras. Em estudos pretéritos de ictioplâncton da baía da Babitonga, a família Sciaenidae é citada entre as mais abundantes, tendo sido registrado, além dos taxa encontrados no presente estudo, o gênero Menticirrhus (Souza-Conceição, 2008; Costa \& Souza-Conceição, 2009).

O padrão de ocorrência temporal das larvas de Sciaenidae na baía da Babitonga já foi observado por Costa \& Souza-Conceição (2009). Maiores densidades foram encontradas durante os meses quentes de primavera e verão, enquanto que nas estações de ou- 
tono e inverno foram verificadas menores densidades. A mesma sazonalidade foi reportada para a Lagoa dos Patos (RS), onde a maior diversidade e abundância de larvas ocorreram no verão (Sinque \& Muelbert, 1998), período de desova de muitas espécies de Sciaenidae (lbagy \& Sinque, 1995).

Espécies de Sciaenidae são encontradas ao longo de todo o estuário da Babitonga, em diferentes estágios de desenvolvimento (Souza-Conceição, 2008; Costa \& Souza-Conceição, 2009; Vilar et al., 2011), sendo que as maiores densidades de larvas foram registradas nos setores externo e intermediário (presente estudo; Souza-Conceição, 2008; Costa \& Souza-Conceição, 2009). Em estudo sobre a utilização de praias estuarinas da baía da Babitonga por larvas e juvenis de peixes, Souza-Conceição (2008) cita Sciaenidae como uma das famílias com o maior número de espécies, sendo $M$. furnieri a mais abundante. Comportamento similar foi observado no estuário da Lagoa dos Patos, com juvenis da espécie ocupando a área estuarina durante todo o ano (Castello, 1986), sendo também encontrada em grandes concentrações na zona de surf da praia adjacente, indicando que os mesmos podem utilizar esse ambiente como área de crescimento (Busoli \& Muelbert, 1999; Busoli \& Muelbert, 2003). Com a desova nas águas costeiras durante o final da primavera e verão, ovos e larvas de $M$. furnieri são transportados para áreas rasas do estuário onde encontram condições favoráveis ao desenvolvimento (Abreu \& Castello, 1998). Assim, para a baía da Babitonga, são necessários estudos com amostras nas demais regiões do estuário, visando compreender de maneira mais completa a distribuição espacial da espécie durante os diferentes estágios de desenvolvimento.

Larvas de $B$. ronchus, as quais tiveram baixa abundância no presente trabalho, foram citadas por Costa \& Souza-Conceição (2009) entre as mais representativas de Sciaenidae para a baía da Babitonga. Johnson (1978) cita o uso de áreas estuarinas com fundos lodosos por juvenis e adultos desse gênero, sendo que a desova da espécie pode ocorrer nestes ecossistemas (Johnson, 1978; Chaves, 1995). I. parvipinnis, $M$. atricauda, $L$. breviceps e $P$. cromis tiveram baixa abundância durante todo o período de estudo, utilizando a baía da Babitonga de forma ocasional.

Os resultados do presente trabalho demonstram que estágios iniciais de Sciaenidae estão fortemente relacionados com o estuário da baía da Babitonga, onde espécies de grande interesse econômico podem ser encontradas durante a fase da vida mais vulnerável. Por outro lado, os ecossistemas estuarinos estão dentre os mais impactados do mundo, o que contribui para a alteração de habitats e mudanças na estrutura e dinâmica das comunidades (Kennish, 2002). Desta for- ma, a importância da conservação de ambientes estuarinos, está associada não só a melhoria da qualidade ambiental, mas também a consequente manutenção de populações de espécies-alvo para a pesca. Neste contexto, a baía da Babitonga, onde foram registrados nove taxa de Sciaenidae em diferentes épocas do ano e estágios de desenvolvimento larval, consiste em um ambiente com status de berçário para espécies comercialmente explotadas no sudeste-sul do Brasil.

\section{AGRADECIMENTOS}

Os autores agradecem o apoio da Universidade do Vale do Itajaí, Universidade da Região de Joinville e Universidade Federal do Paraná. A Coordenação de Aperfeiçoamento de Pessoal de Nível Superior (CAPES) pela concessão da bolsa de mestrado para a primeira autora. Aos Dr. Charrid Resgalla Júnior, Dr. José Angel Alvarez Perez, Dr. José Henrique Muelbert e dois revisores anônimos pela revisão crítica e sugestões para o aprimoramento do trabalho.

\section{REFERÊNCIAS BIBLIOGRÁFICAS}

Abreu, P.C. \& Castello, J.P. 1998. Interações entre os ambientes estuarino e marinho. In: Seeliger, U.; Odebrecht, C. \& Castello, J.P. (Eds). Os ecossistemas marinho e costeiro do extremo sul do Brasil. FURG. 199-203pp.

Babler, S.J.M. 2000. Tropical estuarine fishes: ecology, explotation and conservation. Blackwell Science, $372 p$.

Barletta-Bergan, A.; Barletta, M. \& Saint-Paul, U. 2002. Structure and seasonal of larval fish in the Caeté river estuary in North Brazil. Estuaries, Coastal and Shelf. 54:193-206.

Bastos, G.C. 2006. Atividade pesqueira na baía da Babitonga. In: Cremer, M.J.; Morales, P.R.D. \& Oliveira, T.M.N. (Eds). Diagnóstico Ambiental da baía da Babitonga. Editora UNIVILLE. 200-244pp.

Busoli, R.O. \& Muelbert, J.H. 1999. Composição taxonômica e distribuição do ictioplâncton na zona de arrebentação da praia do Cassino. Atlântica, 21: 19-35.

Busoli, R.O. \& Muelbert, J.H. 2003. Environmental factors and ichthyoplankton dynamics in the surf zone of Cassino sandy beach (32 $\left.{ }^{\circ} 10^{\prime} \mathrm{S}, 52^{\circ} 20^{\prime} \mathrm{W}\right)$. Journal of Coastal Research, SI (35): 463-471.

Boehlert, G.W. \& Mundy, B C. 1988. Roles of behavioral and physical factors in larval and juvenile fish recruitment to estuarine nursery areas. American Fisheries Society Symposium, 3: 51-67. 
Bonecker, F.T.; Castro, M.S. \& Bonecker, A.C.T. 2009. Larval fish assemblage in a tropical estuary in relation to tidal cycles, day/night and seasonal variations. Pan-American Journal of Aquatic Sciences. 4(2): 238-245.

Camacho, F.P. \& Souza-Conceição, J.M. 2007. Distribuição espaço-temporal da clorofila a e das variáveis ambientais em praias estuarinas da ilha de São Francisco do Sul (baía da Babitonga, sul do Brasil). Boletim Técnico-Científico do CEPENE. 15(2): 9-16.

Castello, J.P. 1986. Distribución, crecimiento y maduración sexual de la corvina juvenil (Micropogonias furnieri) en el estuario de la Lagoa dos Patos, Brasil. Physis. 44(106): 21-36.

Cergole, M.C.; Ávila-da-Silva, A.O. \& RossiWongtschowski, C.L.D.B. (Eds). 2005. Análise das principais pescarias comerciais da Região Sudeste-Sul do Brasil: dinâmica populacional das espécies em explotação. Série Documentos REVIZEE, 176p.

Chaves, P.T.C. 1995. Atividade reprodutiva de Bairdiella ronchus (Cuvier) (Pisces, Sciaenidae) na baía de Guaratuba, Paraná, Brasil. Revista Brasileira de Zoologia. 12(4): 759-766.

Clarke, K.R. \& Gorley, R.N. 2006. PRIMER v6: User manual and tutorial. Plymouth Marine Laboratory, $190 p$.

Côrrea, M.F.M.; Pinheiro, P.C.; Almeida, H.K.; Kersten, M.; Lienstadt, J. \& Vollrath, F. 2006. Diagnóstico ambiental da ictiofauna. In: Cremer, M.J.; Morales, P.R.D. \& Oliveira, T.M.N. Diagnóstico Ambiental da baía da Babitonga. Editora UNIVILLE.158-186pp.

Coser, L.M.; Pereira, B.B. \& Joyeux, J.C. 2007. Descrição da comunidade ictioplanctônica e sua distribuição espacial no estuário dos rios Piraquê-Açu e Piraquê-Mirim, Aracruz, ES, Brasil. Interciência. 32(4): 233-241.

Costa, M.D.P. \& Souza-Conceição, J.M. 2009. Composição e abundância de ovos e larvas de peixes na baía da Babitonga, Santa Catarina, Brasil. Pan-American Journal of Aquatic Science. 4(3): 372-382.

Costa, M.D.P.; Souza-Conceição, J.M.; Schwingel, P.R. \& Spach, H. 2011. Assessment of larval distribution of invasive Omobranchus punctatus (Valenciennes, 1836) (Pisces: Blenniidae) in a subtropical estuary (Southern Brazil). Aquatic Invasions, 6(SI): S33-S38.

Diaz, F.; Raimbault, P. \& Conan, P. 2000. Small scale study of primary productivity during spring in a Mediterranean coastal area (Gulf of Lions). Continental Shelf Research, 20: 975-996.

Ditty, J.G. 1989. Separating early larvae of sciaenids from the western North Atlantic : a review and comparison of larvae off Louisiana and Atlantic coast of the U.S. Bulletin of Marine Science. 44(3): 1083-1105.

Elliott, M. \& Hemingway, K.L. 2002. Fishes in estuaries. Blackwell Science, 636p.

Fahay, M.P. 1983. Guide to the early stages of marine fishes occurring in the western north Atlantic ocean, Cape Hatteras to the Southern Scotian Shelf. Journal Northwest Atlantic Fisheries Science, 4: 423p.

Haimovici, M. 1998. Present state and perspectives for the southern Brazil shelf demersal fisheries. Fisheries Management and Ecology. 5: 277-289.

Hoss, D.E. \& Thayer, G.W. 1993. The importance of habitat to the early life history of estuarine dependent fishes. In: Fuiman, L.A. (Ed.). Water quality and the early life stages of fishes. American Fisheries Society Symposium. 14: 147-158.

Ibagy, A.S. \& Sinque, C. 1995. Distribuição de ovos e larvas de Sciaenidae (Perciformes: Teleostei) na região costeira do Rio Grande do Sul - Brasil. Arquivos de Biologia e Tecnologia. 38(1): 249-270.

IBAMA. 1998. Proteção e controle de ecossistemas costeiros manguezal da baía da Babitonga. Instituto Brasileiro do Meio Ambiente. Coleção Meio Ambiente. Série Estudo Pesca, $n^{\circ} 25$. Brasília. $145 p$.

Itagaki, M.K.; Ohkawara, M.H,; Dias, J.F. \& Katsuragawa, M. 2007. Description of larvae and juveniles of Bairdiella ronchus (Sciaenidae: Teleostei) in southeastern Brazil. Scientia Marina. 71(2):249-257.

Johnson, G.D. 1978. Development of fishes of the Atlantic Bight. An atlas of egg, larval, and juvenile stages. IV. Carangidae through Ephipiidae. U.S. Department of the Interior, Fish and Wildlife Service, Biological Services Program. FWS/OBS78/12. 314 p.

Kennish, M.J. 2002. Environmental threats and environmental future of estuaries. Environmental Conservation. 29(1): 78-107.

Leis, J.M. \& Rennis, D.S. 1983. The larvae of IndoPacific coral reef fishes. University of Hawai Press. Hawai. 269p.

Leis, J.M. \& Trnski, T. 1989. The larvae of Indo-Pacific Shorefishes. University of Hawai Press. Hawai. $1^{\circ}$ ed. $371 \mathrm{p}$.

Lenanton, R.C.J. \& Potter, I.C. 1987. Contribution of estuaries to commercial fisheries in temperate western Australia and the concept of estuarine dependence. Estuaries. 10(1): 28-35.

Luczkovich, J.J.; Pullinger, R.C.; Johnson, S.E.; Sprague, M.W. 2008. Identifying sciaenid critical spawning habitats by the use of passive acoustics. 
Transactions of the American Fisheries Society. 137: 576-605.

Machado, E.C.; Daniel, C.B.; Brandini, N. \& Queiroz, R.L.V. 1997. Temporal and spatial dynamics of nutrients and particulate suspended matter in Paranaguá bay, PR, Brazil. Nerítica, 11: 15-34.

Matsuura, Y. \& Nakatani, K. 1979. Ocorrências de larvas e jovens de peixes na ilha de Anchieta (SP), com algumas anotações sobre a morfologia da castanha, Umbrina coroides CUVIER, 1830. Boletim do Instituto Oceanográfico. 28(1): 165-183.

Menezes, N.A. \& Figueiredo, J.L. 1980. Manual de peixes marinhos do sudeste do Brasil. IV. Teleostei (3). Museu de Zoologia USP, 96p.

Moser, H.G. 1996. The early stages of fishes in the California Current Region. California Cooperative Ocean Fisheries Investigations (CALCOFI). ATLAS $N^{\circ}$ 33. Allen Press, Inc., Lawrence, Kansas, USA. $\mathrm{XII}+1505 p$.

Muelbert, J.H. \& Weiss, G. 1991. Abundance and distribution of fish larvae in the channel area of the Patos Lagoon Estuary, Brazil. In: Larval fish recruitment and research in the Americas: proceedings of the $13^{\circ}$ annual fish conference. México. 43-54.

Nelson, J.S. 2006. Fishes of the world. $4^{\text {th }}$ ed. John Wiley \& Sons, 622p.

Olivar, M.P. \& Fortuño, J.M. 1991. Guide to icthyoplankton of the Southeast Atlantic (Benguela Current Region). Scientia Marina. 55(1): 1-383.

Oliveira, T.M.N.; Tureck, C.R.; Bassfeld, J.C.; Torrens, B.M.OI.; Faria, J.M. \& Brasil, K. 2006. Integridade ambiental da baía da Babitonga: características físico-químicas, microbiológicas e ecotoxicidade. In: Cremer, M.J.; Morales, P.R.D. \& Oliveira,
T.M.N. (Eds). Diagnóstico Ambiental da baía da Babitonga. Editora UNIVILLE. 20-80pp.

Ré, P.M.A.B. 1999. Ictioplâncton estuarino da península Ibérica (Guia de Identificação dos ovos e estados larvares planctônicos). Lisboa. 141p.

Richards, W.J. 2006. Early Stages of Atlantic Fishes. An Identification Guide for the Western Central North Atlantic. CRC / Taylor \& Francis, 2 v, 2640p.

Sinque, C. 1980. Larvas de Sciaenidae (Teleostei) identificadas na região estuarino-lagunar de Cananéia. Boletim de Zoologia. 5: 39-77.

Sinque, C. 1989. Ictioplâncton do ecossistema da baía de Paranaguá. Arquivos de Biologia e Tecnologia. 32(3): 473-490.

Sinque, C. \& Muelbert, J.H. 1998. O ambiente e a biota do estuário da Lagoa dos Patos: Ictioplâncton. In: Seeliger, U.; Odebrecht, C. \& Castello, J.P. (Eds). Os ecossistemas costeiro e marinho do sul do Brasil. Editora Ecoscientia. 56-60pp.

Souza-Conceição, J.M. 2008. Praias estuarinas como habitat de criação para estágios iniciais de peixes na ilha de São Francisco do Sul (baía da Babitonga, Santa Catarina). Tese apresentada à Universidade Federal do Paraná. 198p.

Vilar, C.C.; Spach, H.L. \& Santos, L.O. 2011. Fish fauna of Baía da Babitonga (southern Brazil), with remarks on species abundance, ontogenetic stages and conservation status. Zootaxa, 2734: 40-52.

Zar, J.H. 1996. Biostatistical analysis. New Jersey, Prentice Hall. 929p. 